\title{
A NEW VAN FOR THE CARRIAGE OF ARTICLES TO AND FROM THE DISINFECTING STATION.
}

\author{
BY HENRY DRAPER BISHOP, M.D., \\ Medical Officer of Health for Guernsey.
}

THE difficulty in finding some satisfactory form of Carrier Van for the conveyance of clothing to and from the disinfector has hitherto been great, as it has been practically impossible to find a van which could be thoroughly sterilized, and so remove all danger of re-infection of the clothes when disinfected.

Most authorities state that it is necessary to employ two separate vans for this service, one for the carriage of articles to the disinfector, and another for returning them after the process of disinfection.

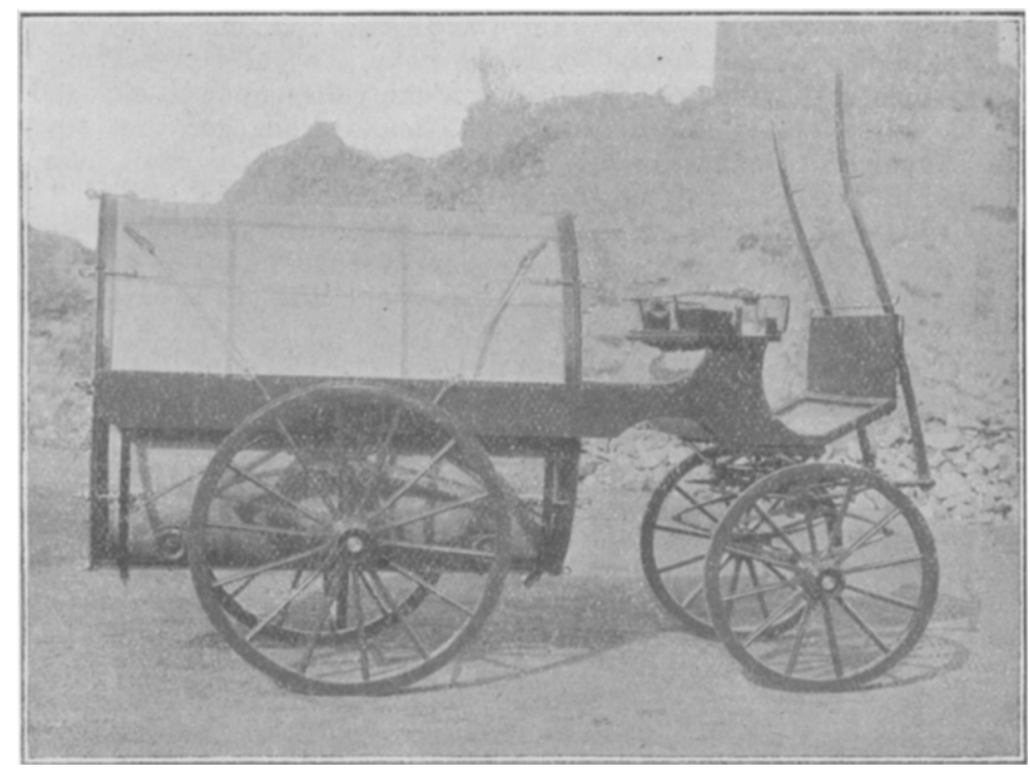

The van under description, which was invented and patented by myself and Mr. A. L. Daris, the manager of the Guernsey Railway Company, is quite free from this objection, and will do the work of the two vans with absolute safety, as, if it be properly worked, re-infection of the disinfected articles is impossible.

The photograph of the existing one, the property of the States of Guernsey, is arranged for use with Messrs, Manlove and Alliotts, Washington Lyon disinfector; but, of course, it could be adapted to suit a disinfector of any other make. The container is made of iron or steel of such shape and size that will just allow of its entrance into the disinfector, and at each end doors are fitted, so arranged that they can be easily removed, at the same time allowing the container to be hermetically sealed, hinged thumb-screws and pin-hinges being convenient for the purpose. The container is mounted on four small 
flanged wheels, and is recessed to receive the wheels as compactly as possible.

The van itself has four wheels, the driving seat accommodating three men, with a space underneath the seat for a sprayer, etc. The axle of the hind wheels is recessed, so as to keep; the container low, the framework consisting of two rails, which support the container by the small flanged wheels, and a strong central beam on each side, which is supported by both ordinary and auxiliary spiral springs, the latter only coming into play when the van is loaded.

On arrival at the disinfecting station, the van is backed against the disinfector on the "infected" side, the rails connected with which are of the same gauge as those of the van, a short length with suitable supports connecting the two.

The doors of the container are removed and placed inside it, and the container is then run into the disinfector, the doors of which are closed, and the container sterilized in the ordinary way.

When the process is complete, the doors of the disinfector are opened on the "uninfected" side, and the container is then run on to the carriage, which has been brought round to receive it.

The van has been found to be light in draft, even in a very hilly district, and to have ample carrying capacity for all ordinary purposes. It is coated with a peculiar variety of paint, which stands the process of disinfection admirably.

It is peculiar in appearance, but not by any means unsightly. If it were thought necessary to disguise its appearance, it could easily be covered with a tarpaulin, or other light and suitable covering. A great point is the simplicity and ease of the process by which the sterilization is accomplished.

\section{WASTE-WATER CLOSETS.}

SINce 1897 all new closet accommodation provided has been upon the water-carriage system, and during the past twelve months the Buildings and Streets Committee have recommended to architects and builders the advisability of putting in separately flushed wash-down w.c.'s in preference to waste-water closets, the latter form of closet having proved so liable to become very foul, and to cause a great waste of water by reason of the water-taps over slop-stones being left runningin some instances all night-for the purpose of flushing the closet. The waste-water closet has also been found to be very liable to become blocked by scrubbing-brushes, scouring-stone and floor-cloths being accidently or carelessly thrown down them, whilst children also make them the receptacle for all manner of refuse, and when so blocked this class of closet is much more difficult and costly to cleanse than an ordinary w.c., by reason of the greater depth of the drain and the difficulty of getting to the trap. Even when properly and carefully used, the tippers become coated with fat and soapy matter, thus being thrown out of balance, and the drain becomes blocked in consequence, whilst the stench arising from the closet pan when hot water is passed through it is, to say the least, very unpleasant. On the other hand, it must be admitted that the waste-water closet is a decided improvement upon the pail or any other conservancy system, whilst it has a slight 